\title{
5-5 Development of an Immersive Teleoperating System for Unmanned Helicopter
}

\author{
Masanao Koeda, * \\ Yoshio Matsumoto ${ }^{\dagger}$ \\ Graduate School of Information Science \\ Nara Institute of Science and Technology \\ CREST, JST(Japan Science and Technology)
}

\author{
Tsukasa Ogasawara ${ }^{\ddagger}$ \\ Graduate School of Information Science \\ Nara Institute of Science and Technology
}

\section{Abstract}

To collect the information on devastated districts, it is effective to use helicopters. However, the operation using manned helicopters costs a lot. It is expected that the use of unmanned helicopters could reduce the cost of these tasks. It is known to be more difficult to operate unmanned helicopters remotely than to operate manned helicopters. The reason is that an operator cannot know the attitude of the helicopter when the operator is far apart from it, and the coordination system between them varies drastically depending on the attitude of the helicopter.

In this paper, we propose an immersive teleoperating method of unmanned helicopters which allows an operator to control unmanned helicopters easily and intuitively as if he is on board. In this method, the operator controls the helicopter remotely while watching the surrounding views of the helicopter through a head mounted display(HMD). To verify the feasibility of the proposed method, we first developed a flight simulator and conducted comparative experiments. Finally we implemented an immersive teleoperating system and conducted an experiment.

\section{Introduction}

Recently unmanned helicopters are used for various purposes, such as crop-dusting and remote sensing. However it is difficult for an operator to control unmanned helicopters remotely. One reason is that an operator cannot be aware of its attitude when the operator is far apart from the helicopter. Another is that the coordination system between the helicopter and the operator changes drastically depending on the attitude of the helicopter. To solve these problems, several studies have been made on autonomous helicopters $[1,2,3]$. However autonomous helicopters need previously determined landmarks or flight paths in order to fly, they are not suitable for flight tasks where the situation changes every minute such as a disaster relief. Additionally, many on-board sensors and computers for controlling are needed. Since the payload of a helicopter is sharply small, autonomous helicopters tend to become large-sized, heavy, and expensive.

There has been a research area called teleoperation or telexistence [4], in which an efficient operation of a remote vehicle is investigated. Telexistence enables a

\footnotetext{
-Address: 8916-5 Takayama-cho, Ikoma, Nara 630-010 Japan. E-mail: masana-k@is.aist-nara.ac.jp

†Address: 8916-5 Takayama-cho, Ikoma, Nara 630-0101 Japan. E-mail: yoshio@is.aist-nara.ac.jp

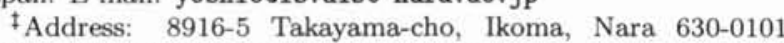
Japan. E-mail: ogasawar@is.aist-nara.ac.jp
}

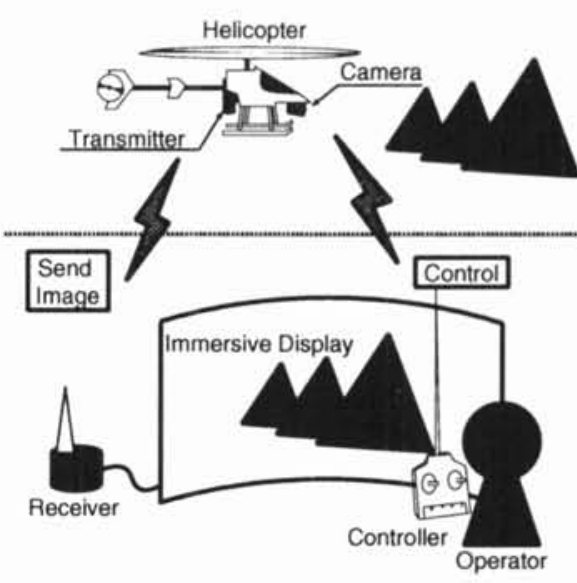

Figure 1: The conceptual diagram of an immersive teleoperating system

human being to have a sensation of existence in a remote environment where a surrogate robot exists.

For instance, Tachi et el.[5] designed and developed telexistence master slave system TELESAR for remote manipulation experiments, and preliminary evaluation experiments of telexistence were conducted for the first time. By comparing the telexistence masterslave system with a conventional system such as observing through a CRT display, the efficacy and the superiority of the telexistence master-slave system were demonstrated experimentally.

Hightower et al. developed a remote presence demonstration system "Greenman." [6] It had an exoskeletal master controller with kinematic equivalency and spatial correspondence of the torso, arms, and head. Its vision system consisted of two video cameras and eyepiece monitors mounted in an aviator's helmet. Even with its simple claw hands and no force or tactile feedback, novice operators were able to perform manipulative tasks without training.

Based on the similar idea to these researches, we propose an immersive teleoperating system for unmanned helicopters.

\section{Immersive Teleoperation of an Un- manned Helicopter}

Figure 1 shows the conceptual diagram of an immersive teleoperating system of an unmanned helicopter. In this system, a camera and a wireless video transmitter are mounted on a helicopter. The captured video image around the helicopter is sent to the operator during the flight. On the ground, a wireless video receiver 


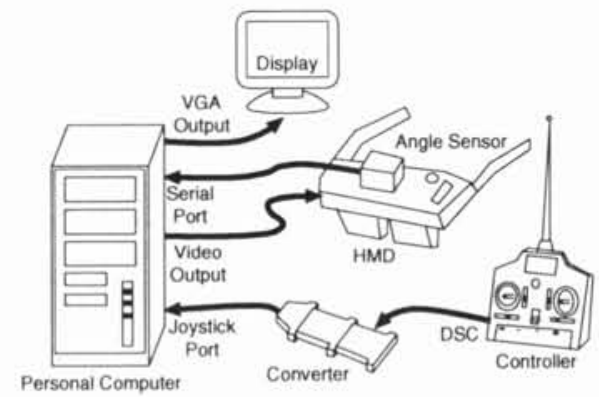

Figure 2: Overview of flight simulator system

picks up the transmitted image and it is displayed on an immersive display. The operator controls the helicopter remotely by watching the surrounding views of the helicopter through the immersive display.

The advantage of this system is that it is necessary to install only a camera and a transmitter on a helicopter. Therefore it is possible to use a compact helicopter with a small payload, and make it light weight and cheap. Additionally, it becomes easy to control an unmanned helicopter because a coordination system between a helicopter and an operator doesn't change even when the attitude of the helicopter changes. Furthermore, an operator can keep controlling even when a helicopter is out of the operator's sight as long as the video image can reach the operator and the helicopter can receive the control signal.

\section{Simulation}

To verify the feasibility of the proposed method, we firstly developed a flight simulator and conducted comparative experiments.

\subsection{System Configuration of Flight Simu- lator}

Figure 2 shows the overview of the flight simulator which we developed for this experiment. This simulator consists of a personal computer, a HMD, an angle sensor, and a controller. An operator wears a HMD with an angle sensor which can measure the orientation of the head. The displayed image on the HMD changes according as the orientation, thus the operator can look around freely. The controller to operate the helicopter in the simulator is the same device as that used for a real radio-controlled helicopter.

This simulator has two modes. We call them "normal flight mode" and "immersive flight mode."

- Normal flight mode(Figure 3)

The operator controls a helicopter wearing a HMD with an angle sensor. In this mode, by heading the direction where he want to see, the operator can look around his circumference as if the operator is standing on the ground.

- Immersive flight mode(Figure 4)

The operator controls a helicopter wearing a HMD with an angle sensor and watchs the image of the camera mounted on helicopter. In this mode, by heading the direction where he want to see, the operator can look around the circumference of the helicopter as if the operator is on board.

\subsection{Experiments with Flight Simulator}

A task was given to five subjects to control the helicopter from a takeoff point to a landing point while

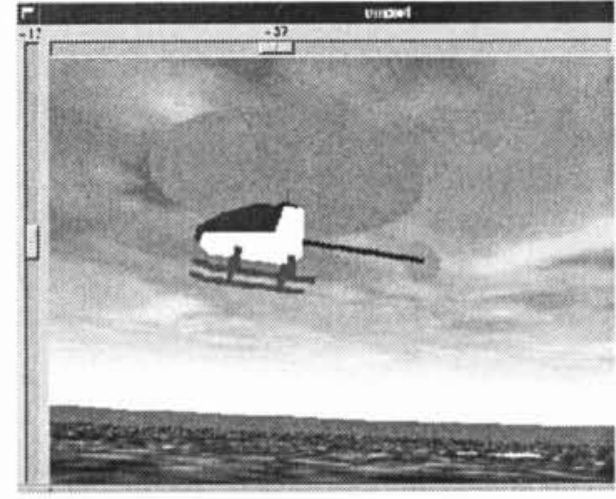

Figure 3: View in normal flight mode

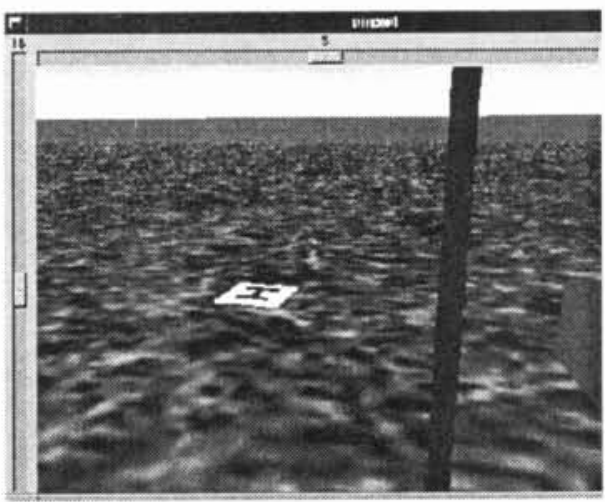

Figure 4: View in immersive flight mode

avoiding an obstacle in the simulator. Figure 5 shows the allocation of the standing point of the operator, the takeoff point, the landing point and the obstacle in the simulation environment. Since all subjects had no experience in operating a radio-controlled helicopter, the experiments were conducted after making practice for 10 minutes in both modes.

Table 1 shows the experimental results in normal flight mode to immersive flight mode. The time to complete and the total flight distance from the takeoff point to the landing point are shown. In terms of flight time, all the subjects completed the task faster using immersive flight mode than using normal flight mode. In terms of total flight distance, three subjects $(A, B$ and $\mathrm{C}$ ) took shorter paths in immersive flight mode. However subject D and E flew for almost the same distance. This is because D and E got accustomed to oparating the helicopter quickly and they were able to take relatively short path in both modes. On the other hand, Subject A, B, and C was unable to operate well

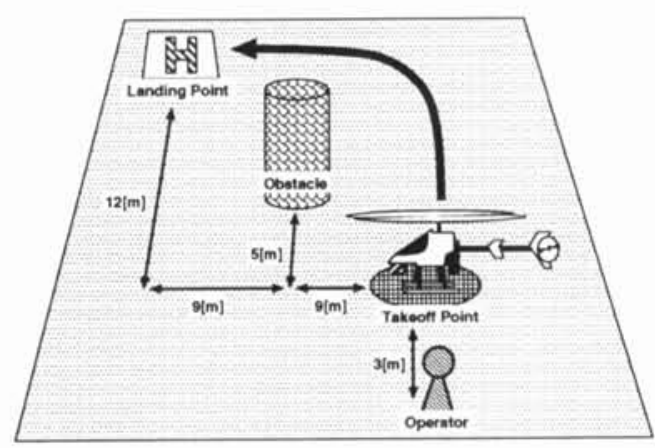

Figure 5: Allocation of takeoff point, landing point, and obstacle in the simulation environment 

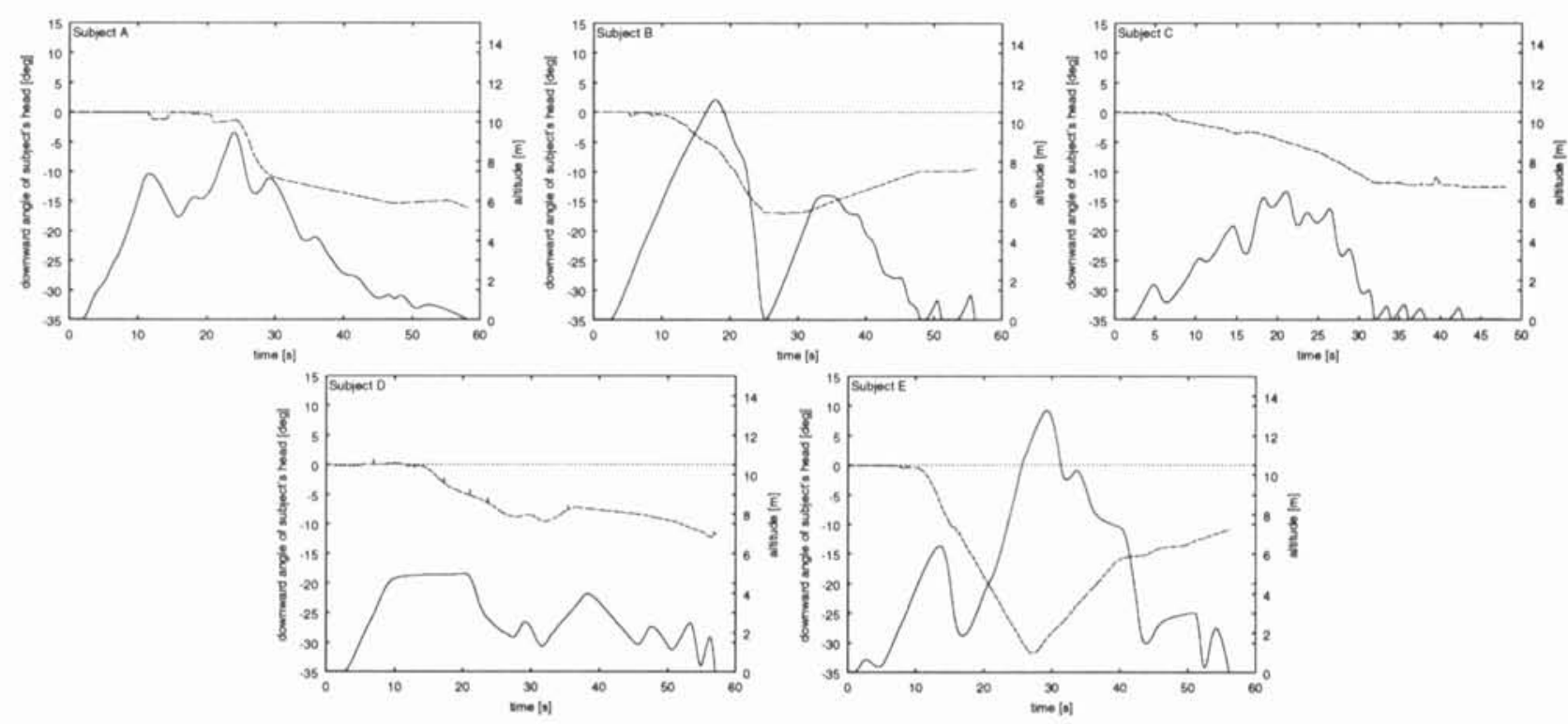

Figure 6: The head attitude of subjects in immersive flight mode

Table 1: Result of experiment using flight simulator

\begin{tabular}{|c|c||c|c|c|c|}
\hline \multicolumn{2}{c|}{} & \multicolumn{2}{c|}{ Time[s] } & \multicolumn{2}{c|}{ Distance[m] } \\
\cline { 3 - 6 } \multicolumn{2}{c|}{} & N & I & N & I \\
\hline \hline \multirow{5}{*}{ Subject } & A & 169 & 56 & 170.4 & 49.1 \\
\cline { 2 - 6 } & B & 84 & 54 & 190.3 & 68.1 \\
\cline { 2 - 6 } & C & 161 & 41 & 307.2 & 43.6 \\
\cline { 2 - 6 } & D & 67 & 54 & 41.0 & 43.1 \\
\cline { 2 - 6 } & E & 96 & 55 & 82.6 & 84.1 \\
\hline
\end{tabular}

$\mathrm{N}$ :Normal flight mode I:Immersive flight Mode

in normal flight mode, since they could not know the attitude of the helicopter when the helicopter was far apart from the operator.

Figure 6 shows the head attitude of subjects in immersive flight mode. In this figure, solid lines indicate the altitude of the helicopter and dashed lines indicate the downward angle of the subject's head. It can be seen from this figure that all of the subjects looked downward to check the landing point before landing the helicopter. This result indicates that the subjects used an immersive teleoperating system effectively.

\section{Implementation}

\subsection{System Configuration}

Figure 7 and 8 illustrate the overview of the developed immersive teleoperating system, and Table 2 shows the specification of this system. In this system, an omnidirectional camera[7] and a wireless video transmitter are mounted at the bottom of the helicopter. The omnidirectional image is sent by the transmitter. Using a hyperboloidal mirror, it is possible to convert an omnidirectional image to perspective images(Figure 9). Since panning and tilting of the camera can be simulated by generating view-dependent images from omnidirectional images, there is no need to attach mechanisms to rotate the camera on the helicopter.

The system on the ground consists of a note PC, a wireless video receiver, a HMD and an angle sensor. A received omnidirectional image is captured by the $\mathrm{PC}$ and converted into a perspective image. Then the converted image is displayed on the HMD which the operator wears. The angle sensor attached to the HMD is utilized to measure the head attitude of the operator. The displayed image to the HMD changes depending

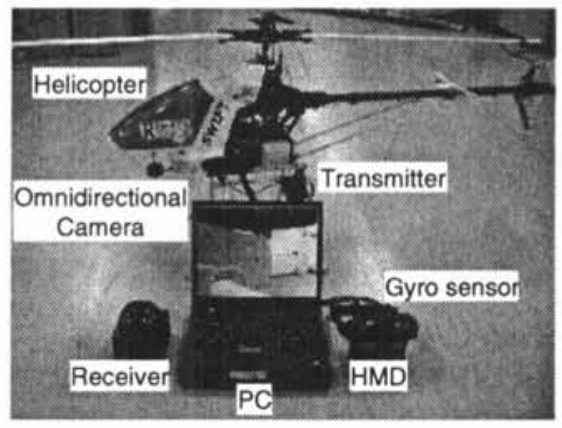

Figure 7: Developed immersive teleoperating system

on the head attitude of the operator, and the operator can freely look around the surrounding views of the helicopter as if he/she is onboard. As a result, it is possible for the operator to control a helicopter with high immersion.

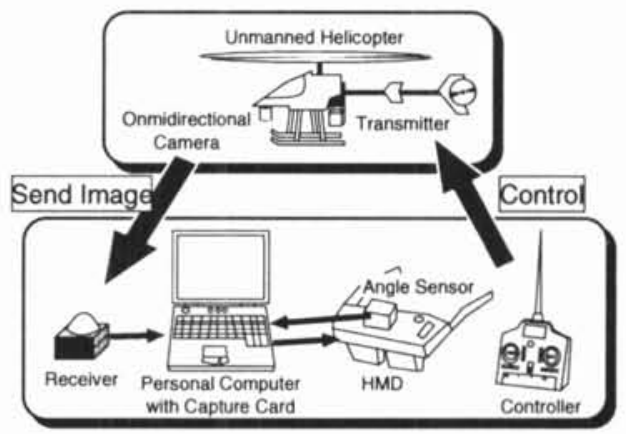

Figure 8: Overview of developed immersive teleoperating system

\subsection{Generating Perspective Images}

Figure 10 shows the geometry of the hyperboloidal mirror "HyperOmni Vision." The projection from the point $p(x, y)$ on the perspective plane to the point $P(X, Y, Z)$ in space is given by

$$
x=\frac{f\left(b^{2}-c^{2}\right) X}{\left(b^{2}+c^{2}\right) Z-2 b c \sqrt{X^{2}+Y^{2}+Z^{2}}}
$$



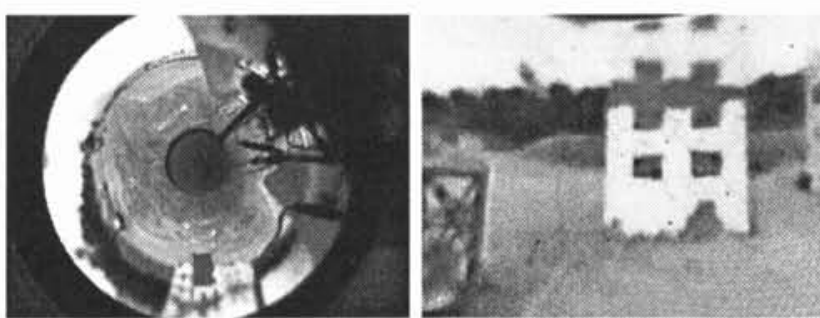

Figure 9: Received omnidirectional image and generated perspective image

Table 2: Specification of developed system

\begin{tabular}{|c|c|}
\hline Helicopter & $\begin{array}{l}\text { HIROBO TURUGI XX } \\
\text { Length: } 1.33[\mathrm{~m}] \\
\text { Height: } 0.64[\mathrm{~m}] \\
\text { Payload: } 1.2[\mathrm{~kg}]\end{array}$ \\
\hline Camera & $\begin{array}{l}\text { ACCOWLE } \\
\text { Omnidirectional Vision Sensor } \\
\text { (Hyperboloidal Mirror) } \\
\text { Resolution: } 512 \times 492[\text { pixel] }\end{array}$ \\
\hline Transmitter & $\begin{array}{l}\text { RF BS-550GTH } \\
\text { Weight: } 0.25[\mathrm{~kg}]\end{array}$ \\
\hline $\mathrm{PC}$ & $\begin{array}{l}\text { OS: Linux } \\
\text { CPU: PentiumIII } 1000[\mathrm{MHz}] \\
\text { Memory: } 384[\mathrm{MB}]\end{array}$ \\
\hline Receiver & RF BS-120GRH \\
\hline HMD & $\begin{array}{l}\text { i-O DisplaySystems i-glasses! LC } \\
\text { Resolution: } 450 \times 266[\text { pixel] }\end{array}$ \\
\hline $\begin{array}{l}\text { Angle } \\
\text { Sensor }\end{array}$ & $\begin{array}{l}\text { INTERSENSE InterTrax } 2 \\
\text { Angular Range: } \\
\text { Pitch: } \pm 80^{\circ}, \text { Yaw: } \pm 180^{\circ}, \text { Roll: } \pm 90^{\circ}\end{array}$ \\
\hline
\end{tabular}

$$
y=\frac{f\left(b^{2}-c^{2}\right) Y}{\left(b^{2}+c^{2}\right) Z-2 b c \sqrt{X^{2}+Y^{2}+Z^{2}}}
$$

where $b, c$ are the parameter of the hyperboloidal mirror and $f$ is the focal point of the camera. By computing above geometric transformation between $(x, y)$ and $(X, Y, Z)$, a perspective image viewing an arbitrary direction from the focal point can be generated. Real-time image transformation was achieved by making lookup tables every $2[\mathrm{deg}]$ for the horizontal head rotation.

\subsection{Experiments with Developed System}

In order to verify the feasibility of this system, we conduct an experiment to operate an unmmand helicopter around the building using the developed system.

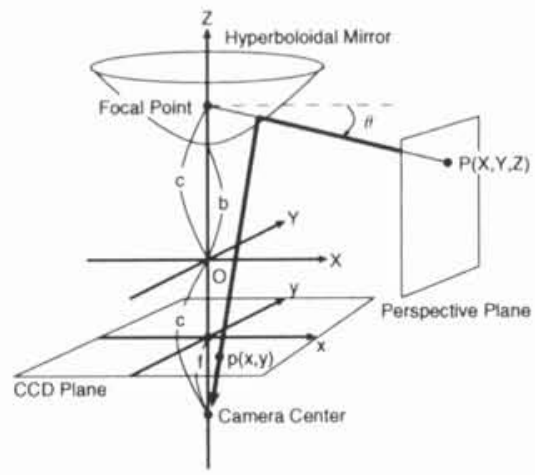

Figure 10: Geometry of HyperOmni Vision
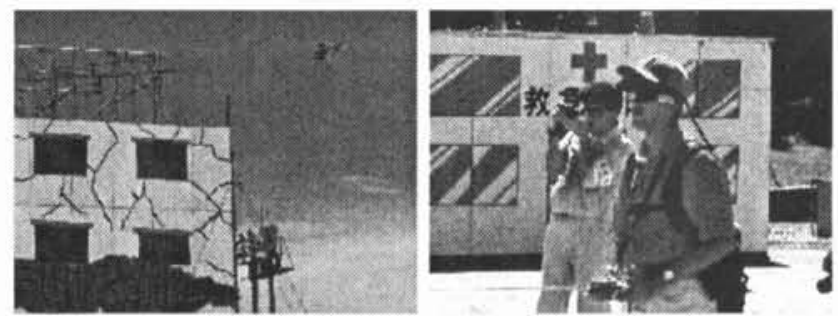

Figure 11: Operation of an unmmand helicopter using developed system

The photographs of the experiment is shown in Figure 11. The operator controlled the unmmand helicopter while looking at the image displayed on the HMD. We confirmed that it was possible to control the helicopter in the situation that the operator could not see it directly. This is the advantage of the developed system compared with the conventional operating method.

\section{Conclusions}

In this paper, we proposed an immersive teleoperating method of an unmanned helicopter which allows an operator to control it easily. To verify the feasibility of the proposed method, we developed a flight simulator and conducted comparative experiments. The experimental results with flight simulator indicate our proposed method has an advantage over the conventional operating method. Then we developed an immersive teleoperating system of an unmanned helicopter which consists of an omnidirectional camera and a HMD with an angle sensor. We finally confirmed the feasibility of the system through real experiments using the developed system.

\section{Acknowledgement}

This research is partly supported by Core Research for Evolutional Science and Technology(CREST) Program "Advanced Media Technology for Everyday Living" of Japan Science and Technology(JST)

\section{References}

[1] Ryan Miller, Omead Amidi, and Mark Delouis: "Arctic Test Flights of the CMU Autonomous Helicopter", Proceeding of the Association for Unmanned Vehicle Systems International 1999 .

[2] Harbick, K., Montgomery, J.F., Sukhatme, G.S.: "Planar Spline Trajectory Following for an Autonomous Helicopter", 2001 IEEE International Symposium on Computational Intelligence in Robotics and Automation.

[3] T. J. Koo, D. H. Shim, O. Shakernia, B. Sinopoli, Y. Ma, F. Hoffmann, S. Sastry: "Hierarchical Hybrid System Design on Berkeley UAV", Submitted to International Aerial Robotics Competition, Richland, Washington, USA, August 1998 .

[4] S. Tachi, K. Tanie, K. Komoriya and M. Kaneko: "Teleexistence(I) -Design and Evaluation of a Visual Display with Sensation of Presence-", Proc. of the 5th Symposium on Theory and Practice of Robots and Manipulators, pp. 245-254, 1984.

[5] S. Tachi and K. Yasude: "Evaluation Experiments of a Tele-existence Manipulation System", Presence, vol. 3, No. 1, pp. $35-44,1994$.

[6] J.D.Hightower, D.C. Smith, and S.F. Wiker: "Development of Remote Presence Technology for Teleoperator Systems", 14th Meeting of the United States-Japan Natural Resources Committee, 1986.

[7] K. Yamazawa, Y. Yagi, and M. Yachida: "New real-time omnidirectional image sensor with hyperboloidal mirror", Proc. 8th Scandinavian Conf. on Image Analysis, Vol. 2, pp. 1381-1387, Tromso, Norway, May 1993. 